\title{
Echocardiographic signs of pulmonary hypertension in patients with newly recognized hypersensitivity pneumonitis, prevalence and clinical predictors
}

\author{
Małgorzata Dybowska ${ }^{1}$, Inga Barańska ${ }^{2}$, Monika Franczuk ${ }^{3}$, Agnieszka Skoczylas $^{4}$, Monika Szturmowicz $^{1}$ \\ ${ }^{1}$ Ist Department of Lung Diseases, National Tuberculosis and Lung Diseases Research Institute, Warsaw, Poland; ${ }^{2}$ Department of Radiology, \\ National Tuberculosis and Lung Diseases Research Institute, Warsaw, Poland; ${ }^{3}$ Department of Physiopathology, National Tuberculosis and Lung \\ Diseases Research Institute, Warsaw, Poland; ${ }^{4}$ Department of Geriatrics, National Institute of Geriatrics, Rheumatology and Rehabilitation, Warsaw, \\ Poland \\ Contributions: (I) Conception and design: M Szturmowicz, M Dybowska; (II) Administrative support: M Szturmowicz, M Dybowska; (III) Provision of \\ study materials or patients: M Dybowska, I Barańska; (IV) Collection and assembly of data: M Franczuk, I Barańska, M Dybowska; (V) Data analysis \\ and interpretation: M Szturmowicz, A Skoczylas; (VI) Manuscript writing: All authors; (VII) Final approval of manuscript: All authors.
}

Correspondence to: Małgorzata Dybowska. 01-138 Warsaw, Poland. Email: dybowska@mp.pl.

Background: Hypersensitivity pneumonitis (HP) is the third, according to frequency, interstitial lung disease, with the estimated incidence rate of 1-2/100,000. In HP patients, the extensive inflammatory lesions encompassing both small airways and lung parenchyma, as well as subsequent development of lung fibrosis, may result in respiratory insufficiency and secondary pulmonary hypertension (PH). The aim of the present retrospective study was to assess the prevalence of echocardiographic signs of $\mathrm{PH}$ and its' clinical predictors, in newly recognized HP patients.

Methods: Consecutive HP patients, recognized in single pulmonary unit between 2005 and 2017, in whom echocardiography was performed at diagnosis, entered the present study. HP diagnosis was verified in every patient according to current diagnostic recommendations. The results of high resolution computed tomography of the chest (HRCT) were re-evaluated by two independent radiologists, blinded to clinical data. Echocardiographic signs of PH were defined as pulmonary artery systolic pressure (PASP) exceeding $36 \mathrm{mmHg}$. Regression analysis was applied to calculate $\mathrm{PH}$ risk, and receiver operator characteristic curves (ROC) were plotted to investigate diagnostic utility of various parameters in $\mathrm{PH}$ prediction.

Results: PASP exceeding $36 \mathrm{mmHg}$ was noted in 26 out of 70 patients (37\%)—with equal frequency among patients with fibrotic and non-fibrotic HP. Significant predictors of $\mathrm{PH}$ on echocardiography were: partial oxygen tension in arterialized capillary blood $(\mathrm{PaO} 2)<69 \mathrm{mmHg}$, lung transfer capacity for carbon monoxide (TLCO) $<42 \%$ of predicted, six minutes walking test (6MWT) distance $<455$ meters, and 6 MWT desaturation rate $>8 \%$. In case of TLCO $<42 \%$ of predicted, probability of $\mathrm{PH}$ on echocardiography was increased by five-fold, in case of $6 \mathrm{MWT}$ desaturation rate $>8 \%$ - by four fold.

Conclusions: The best predictors of PASP $>36 \mathrm{mmHg}$ on echocardiography in HP patients at diagnosis were: TLCO $<42 \%$ and $6 \mathrm{MWT}$ desaturation rate $>8 \%$. Neither the presence of lung fibrosis on HRCT, nor the duration of the disease or patients age, were helpful in $\mathrm{PH}$ prediction.

Keywords: Hypersensitivity pneumonitis (HP); pulmonary hypertension (PH); lung transfer capacity for carbon monoxide (TLCO)

Submitted Jan 21, 2021. Accepted for publication May 20, 2021.

doi: $10.21037 /$ jtd-21-130

View this article at: https://dx.doi.org/10.21037/jtd-21-130

(c) Journal of Thoracic Disease. All rights reserved. 


\section{Introduction}

Hypersensitivity pneumonitis (HP) belongs to the group of interstitial lung diseases with known etiologic factors. The environmental, occupational or hobbyist exposition to organic antigens induces the immunological response, leading in genetically predisposed individuals to chronic inflammatory lung disease (1). Lung biopsy specimens typical of HP, show the features of cellular, bronchiolocentric, lymphocyte - predominant interstitial pneumonia and bronchiolitis, with the presence of poorly formed non-necrotizing granulomas (2). In some patients, chronic inflammation progresses to fibrotic lung disease, with airway centred fibrosis and characteristic bridging fibrosis (2).

Although, HP was previously regarded a rare ILD, nowadays it is usually listed on the third position among interstitial lung diseases, after idiopathic pulmonary fibrosis (IPF) and ILDs in the course of connective tissue diseases or sarcoidosis $(3,4)$.

Recent data indicate growing recognition of HP in many countries, the actual incidence rate is estimated at approximately $1-2 / 100,000(5,6)$.

The diagnostic criteria of HP that have been published recently, include: pulmonary symptoms related to the exposition to responsible antigens and/or the presence of specific serum IgG antibodies (ssIgGs), typical picture of high resolution computed tomography of the chest (HRCT), and increased ( $>30 \%$ ) percentage of lymphocytes in bronchoalveolar lavage fluid (BALF) (2). Final diagnosis should be supported by the result of multidisciplinary discussion. Transbronchial lung biopsy (TBLB) is suggested as the procedure of first choice, in the patients with nonfibrotic lung disease (2). In case of diagnostic difficulties, the second line procedures, such as transbronchial cryobiopsy or surgical lung biopsy, may be recommended by multidisciplinary panel to ascertain the diagnosis (2).

Current guidelines distinguish two forms of HP, fibrotic and non-fibrotic (2). The terms acute HP and chronic HP have been abandoned, due to less clear prognostic significance (2).

Pulmonary hypertension $(\mathrm{PH})$ is one of the negative prognostic factors in many end-stage lung diseases. The knowledge about incidence of $\mathrm{PH}$ in $\mathrm{HP}$, especially at diagnosis, is scarce. Moreover, it is not known if there is any clinical phenotype of HP suggestive for $\mathrm{PH}$ on echocardiographic examination.

Therefore, the aim of the present retrospective study was to assess the prevalence of echocardiographic signs of $\mathrm{PH}$ and its' clinical predictors, in newly recognized HP patients.

We present the following article in accordance with the STARD reporting checklist (available at https://dx.doi. org/10.21037/jtd-21-130).

\section{Methods}

Consecutive patients with HP diagnosed between 2005 and 2017 in the single pulmonary unit, in whom 2D-Doppler echocardiographic transthoracic examination was performed at diagnosis, entered the present study.

The algorithm of HP recognition was based on: positive exposure to organic antigens and/or positive ssIgGs, typical HRCT pattern and increased lymphocytosis in BALF, exceeding $30 \%$. In case of unclear diagnosis, transbronchial lung biopsy or surgical lung biopsy were performed. The detailed information concerning the applied diagnostic procedures has been published previously $(7,8)$.

2D-Doppler echocardiographic transthoracic examination, was performed with Siemens Acuson, Sequoia, or Toshiba Medical Systems, SSH-880 CV/W1 Artida. Two-dimensional and colour-flow guided continuous-wave Doppler transthoracic echocadiography was performed by experienced cardiac sonographers according to current standards. Pulmonary artery pressure was estimated based on the peak tricuspid regurgitation velocity (TRV). The tricuspid regurgitation pressure gradient (TRPG, TVPG) was calculated according to the modified Bernoulli equation: TRPG $=4 \times(T R V)^{2}$ and systolic PAP (PASP) was calculated from the equation: $\mathrm{PASP}=\mathrm{TRPG}+$ estimated right atrial pressure. Probability of $\mathrm{PH}$ was defined according to 2009 ESC/ERS guidelines, as most patients have been examined at that period of time (9). According to PASP results the patients were divided into two groups:

(I) $\mathrm{PH}$ unlikely: TRV $\leq 2.8 \mathrm{~m} / \mathrm{s}$, PASP $\leq 36 \mathrm{mmHg}$,

(II) $\mathrm{PH}$ possible or likely TRV $2.9-3.4 \mathrm{~m} / \mathrm{s}$, PASP $37-50 \mathrm{mmHg}$ or TRV $>3.4 \mathrm{~m} / \mathrm{s}$, PASP $>50 \mathrm{mmHg}$.

Additionally, pulsed-wave Doppler was used to measure pulmonary artery acceleration time (AcT), defined as the interval between the onset of systolic pulmonary arterial flow and peak flow velocity.

The results of HRCT were re-evaluated retrospectively by two independent radiologists, blinded to clinical data. For the purposes of CT scan analyses, each lung was divided into the upper, central, and lower fields. From each lung field a transverse scan with the highest intensity of lesions was chosen in accordance by two radiologists. Qualitative 
and quantitative assessment was performed within the lung window using a $0-1-2$ scale to evaluate pulmonary lesions (ill-defined centrilobular nodules, ground glass opacities, mosaic attenuation and/or air trapping). $0=$ no radiological signs in both lungs, $1=$ the changes identified in $1-3$ lung fields, $2=$ the changes identified in $4-6$ lung fields. Degree of lung fibrosis was coded as: $0=$ no fibrosis, $1=$ reticular and/or bronchiectatic fibrosis, $2=$ honeycombing.

Plethysmography was performed with Master Screen Body/Diffusion by Jaeger (Germany 2002), according to ERS/ATS recommendations (10). The values of pulmonary function indices were reported as percentages of predicted values, according to ERS reference equations (11). For FEV1 and FVC - Falaschetti reference equations have been used (12). Lung transfer capacity for carbon monoxide (TLCO), was examined with the single breath method, using helium gas as a marker (13). The results were expressed as a percentage of predicted values, with correction for haemoglobin.

Six minutes walking test (6MWT) was performed on a corridor, with baseline and sixth minute room air oxygen saturation, according to ATS guidelines (14).

Serum N-terminal pro-B-type natriuretic peptide (NTproBNP) was assessed with Elecsys proBNP II, Cobas e411, Roche Diagnostics GmbH, Germany.

The study was conducted in accordance with the Declaration of Helsinki (as revised in 2013). The study was approved by Bioethical Committee, National Tuberculosis and Lung Diseases Research Institute (NP.: 14/2019) and informed consent was taken from all the patients.

\section{Statistical analysis}

All analyses were performed with $\mathrm{R}$ version 3.6.0-a software environment for statistical computing and graphics (https://www.r-project.org/). Continuous variables were presented as means and standard deviations, categorical ones-as percentages of the entire population studied. Distributions' normality and homogeneity of variance of continuous variables in different groups were checked with Shapiro-Wilk test and F test, respectively. T-Student test (T-St) or U Mann-Whitney test $(\mathrm{M}-\mathrm{W})$, were used for comparison of the two groups. Categorical variables distribution was compared with Pearson's test, with its modifications if applicable. Correlations were analysed with Spearman's rank order test or Pearson's test.

Youden method was used to calculate cut off values of parameters with the highest specificity and sensitivity for echocardiographic $\mathrm{PH}$ prediction. Regression analysis (odds ratios and $95 \%$ confidence intervals) was applied to assess the best predictors of $\mathrm{PH}$ on echocardiography. A receiver operator characteristic curves were plotted for selected predictive parameters.

\section{Results}

Seventy patients with newly recognized HP entered the study. The population characteristics are presented in Table 1.

In 26 out of 70 patients ( $37 \%)$, PASP exceeding $36 \mathrm{mmHg}$ was noted, in 5 of 70 (7\%) PASP exceeded $50 \mathrm{mmHg}$.

RV was significantly larger and AcT significantly shorter in the patients with PASP $>36 \mathrm{mmHg}$, comparing to those with PASP $\leq 36 \mathrm{mmHg}$ (Table 2).

Demographic and clinical data as well as plethysmography, TLCO and walking test results in the groups of patients with PASP $>36 \mathrm{mmHg}$ and with PASP $\leq 36 \mathrm{mmHg}$ were compared in Table 3 .

6MWT walking distance (6MWD) was significantly shorter in the patients with PASP $>36 \mathrm{mmHg}$, comparing to those with PASP $\leq 36 \mathrm{mmHg}$ (mean values 441.23 meters and 511.98 meters, respectively, $\mathrm{P}=0.006$ ), and desaturation rate on exertion - significantly higher (mean values $11.08 \%$ and $5.77 \%$ respectively, $\mathrm{P}=0.001$ ).

Significant correlations were found between TVPG and 6MWD (Figure 1), as well as between TVPG and desaturation rate on exertion (Figure 2).

Analysis of respiratory indices revealed significantly lower TLCO \% pred. in patients with PASP $>36 \mathrm{mmHg}$, comparing to those with PASP $\leq 36 \mathrm{mmHg}$, (mean values: $41.08 \%$ and $52.64 \%$, respectively, $\mathrm{P}=0.002$ ). The correlation between TVPG and TLCO \% pred. is presented on Figure 3.

Arterialised capillary blood gas analysis revealed significantly lower partial oxygen tension $\left(\mathrm{PaO}_{2}\right)$ in patients with PASP $>36 \mathrm{mmHg}$, comparing to the remaining ones (mean values: 62 and $72.68 \mathrm{mmHg}$, respectively, $\mathrm{P}=0.0002$ ). The correlation between TVPG and $\mathrm{PaO}_{2}$ is presented on Figure 4.

The prevalence of fibrotic HP on HRCT was equal in patients with PASP $>36 \mathrm{mmHg}$ and those with PASP $\leq 36 \mathrm{mmHg}$ ( $66 \%$ and $61 \%$, respectively) (Table 4 ). Nevertheless, in 5 patients with PASP $>50 \mathrm{mmHg}$-all presented with lung fibrosis: stage 1, in 2 cases; stage 2, in 3 cases. The presence and extension of ground glass 
Table 1 Characteristics of study population

\begin{tabular}{|c|c|}
\hline Variables & Mean \pm SD \\
\hline Age at diagnosis (years) & $52.63 \pm 11.75$ \\
\hline Males/females (No.) & $35 / 35$ \\
\hline Duration of symptomatic disease (months) & $34.04 \pm 40.49$ \\
\hline \multicolumn{2}{|l|}{ Exposure to No. (\%) } \\
\hline Hay and products & $32[46]$ \\
\hline Poultry & $22[31]$ \\
\hline Pigeons & 15 [21] \\
\hline Others & $18[26]$ \\
\hline \multicolumn{2}{|l|}{ HRCT } \\
\hline Fibrotic HP & $45[64]$ \\
\hline Non-fibrotic HP & $25[36]$ \\
\hline TLC (\% pred) & $88.58 \pm 23.54$ \\
\hline VC (\% pred) & $81.52 \pm 20.8$ \\
\hline TLCO (\% pred) & $48.34 \pm 34$ \\
\hline $\mathrm{PaO}_{2}(\mathrm{mmHg})$ & $68.7 \pm 11.7$ \\
\hline $\mathrm{PaCO}_{2}(\mathrm{mmHg})$ & $36.67 \pm 67$ \\
\hline 6MWD (meters) & $485.7 \pm 106.3$ \\
\hline 6MWT desaturation (\%) & $7.7 \pm 7.07$ \\
\hline NT-proBNP (pg/mL) & $139.53 \pm 296.49$ \\
\hline
\end{tabular}

HRCT, high resolution computed tomography of the chest; HP, hypersensitivity pneumonitis; TLC, total lung capacity; VC, vital capacity; TLCO, lung transfer capacity for carbon monoxide; $\mathrm{PaO}_{2}$, partial oxygen tension measured in arterialized capillary blood; $\mathrm{PaCO}_{2}$, carbon dioxide tension measured in arterialized capillary blood; 6MWT, six minutes walking test; 6MWD, six minutes walking test distance; NT-proBNP, serum N-terminal pro-B-type natriuretic peptide; SD, standard deviation. opacities, ill-defined centrilobular nodules and mosaic lung attenuation pattern on HRCT were not helpful in predicting $\mathrm{PH}$ on echocardiography.

According to ROC analysis, the highest diagnostic utility for prediction of $\mathrm{PASP}>36 \mathrm{mmHg}$ was combined with $\mathrm{PaO}_{2}$ $<69 \mathrm{mmHg}$, TLCO $<42 \%$ pred., $6 \mathrm{MWD}<455$ meters, and $6 \mathrm{MWT}$ desaturation rate $>8 \%$ (Table 5, Figure 5).

Univariate analysis, documented that all the above mentioned parameters were the significant predictors of PASP $>36 \mathrm{mmHg}$ (Table 6). Risk of $\mathrm{PH}$ on echo was increased five-fold in case of TLCO $<42 \%$ pred., fourfold-in case of exercise desaturation $>8 \%$, three-fold-in case of $6 \mathrm{MWD}<455$ meters and two-fold-in those with $\mathrm{PaO}_{2}<69 \mathrm{mmHg}$.

\section{Discussion}

Echocardiography is considered the best non-invasive method to screen for $\mathrm{PH}$ associated with lung diseases (15). Symptomatic patients with high probability of $\mathrm{PH}$ on echocardiography, should be considered as candidates to right heart catheterization (RHC), especially when their future management would be influenced by RHC results (15). RHC is particularly indicated in case of: referral for lung transplantation, inclusion in clinical trials or planned PH-dedicated therapy (15). Nevertheless, most patients with ILD at the time of diagnosis, would not profit from RHC, and thus the accepted method of $\mathrm{PH}$ evaluation is echocardiography.

Majority of published data documenting $\mathrm{PH}$ prevalence in the early period of interstitial lung diseases, concern sarcoidosis and IPF. In PULSAR study, based on echocardiography and $\mathrm{RHC}, \mathrm{PH}$ was found in $3 \%$ of

Table 2 Echocardiographic data in HP patients with signs of $\mathrm{PH}$ comparing to those without $\mathrm{PH}$

\begin{tabular}{lccc}
\hline Parameter & PASP $>36 \mathrm{mmHg}$ & PASP $\leq 36 \mathrm{mmHg}$ & P \\
\hline TVPG $(\mathrm{mmHg})$ & $40.22 \pm 10.07$ & $24.66 \pm 4.1$ & 0.000000002 \\
AcT $(\mathrm{ms})$ & $80.48 \pm 9.74$ & $117.3 \pm 21.44$ & 0.000000002 \\
RV $(\mathrm{mm})$ & $27.26 \pm 3.55$ & $25.07 \pm 4.32$ & 0.03 \\
LV $(\mathrm{mm})$ & $45.56 \pm 5.03$ & $46.09 \pm 5.69$ & 0.69 \\
\hline
\end{tabular}

HP, hypersensitivity pneumonitis; PH, pulmonary hypertension; PASP, pulmonary artery systolic pressure; TVPG, trans-tricuspid valve gradient; AcT, pulmonary artery acceleration time; RV, right ventricle diameter; LV, left ventricle diameter; M-W, U Mann-Whitney test; T-St, T Student test. 
Table 3 Clinical data, plethysmography results, 6MWT, NT-proBNP and arterialized capillary blood gas analysis in HP patients with echocardiographic signs of $\mathrm{PH}$ comparing to those without echocardiographic signs of $\mathrm{PH}$

\begin{tabular}{|c|c|c|c|c|}
\hline Parameter & $\mathrm{PASP}>36 \mathrm{mmHg}$ & PASP $\leq 36 \mathrm{mmHg}$ & $\mathrm{P}$ & Test type \\
\hline Age at diagnosis (years) & $55.56 \pm 9.22$ & $50.84 \pm 12.83$ & 0.1 & T-St \\
\hline TLC (\% pred) & $81.81 \pm 24.05$ & $92.93 \pm 22.43$ & 0.055 & T-St \\
\hline VC (\% pred) & $74.04 \pm 19.14$ & $86.11 \pm 20.65$ & 0.016 & T-St \\
\hline TLC\%/TLCO\% & $2.26 \pm 0.86$ & $1.79 \pm 0.52$ & 0.008 & $M-W$ \\
\hline VC\%/TLCO\% & $2.09 \pm 0.8$ & $1.72 \pm 0.54$ & 0.06 & $M-W$ \\
\hline $\mathrm{PaO}_{2}(\mathrm{mmHg})$ & $62 \pm 8.82$ & $72.68 \pm 11.88$ & 0.0002 & T-St \\
\hline $\mathrm{PaCO}_{2}(\mathrm{mmHg})$ & $37.5 \pm 3.91$ & $36.18 \pm 4.51$ & 0.22 & T-St \\
\hline NT-proBNP (pg/mL) & $226.43 \pm 449.1$ & $85.2 \pm 114.36$ & 0.23 & $M-W$ \\
\hline
\end{tabular}

6MWT, six minutes walking test; NT-proBNP, serum N-terminal pro-B-type natriuretic peptide; HP, hypersensitivity pneumonitis; PH, pulmonary hypertension; PASP, pulmonary artery systolic pressure; TLC, total lung capacity; VC, vital capacity; TLCO, lung transfer capacity for carbon monoxide; $\mathrm{PaO}_{2}$, partial oxygen tension measured in arterialized capillary blood; $\mathrm{PaCO}$, $\mathrm{Carbon}$ dioxide tension measured in arterialized capillary blood; 6MWD, six minutes walking test distance; M-W, U Mann-Whitney test; T-St, T Student test.

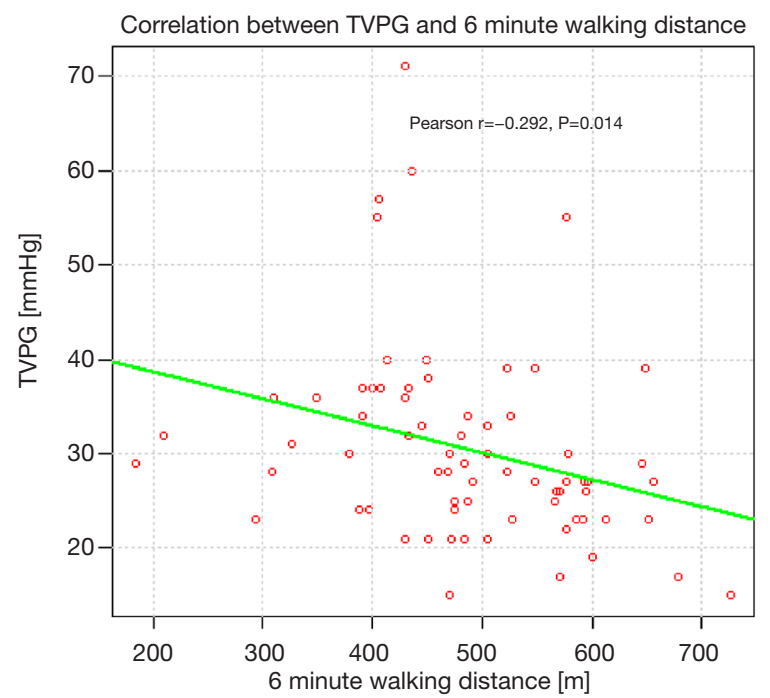

Figure 1 Correlation between TVPG and 6MWD in $70 \mathrm{HP}$ patients. $6 \mathrm{MWD}$, six minutes walking distance; $\mathrm{HP}$, hypersensitivity pneumonitis.

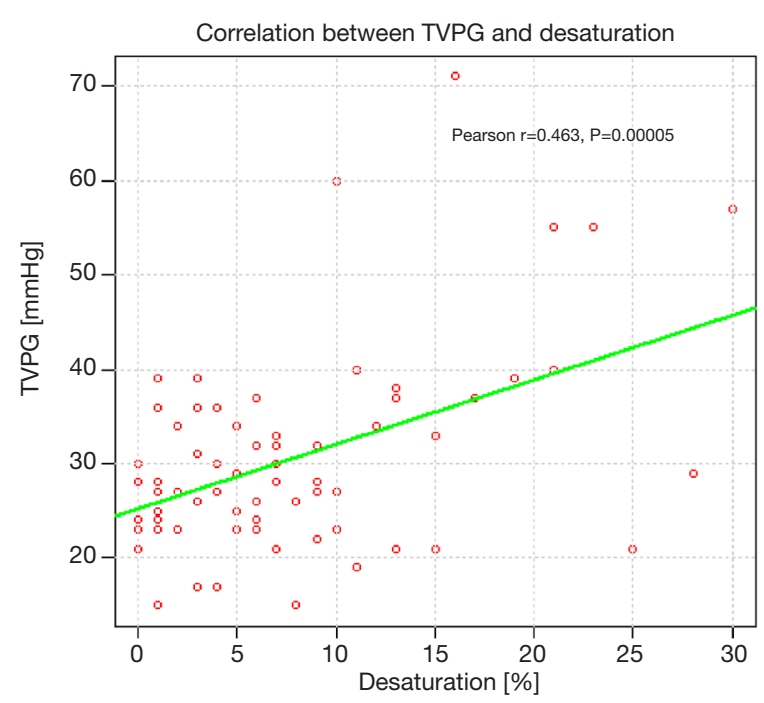

Figure 2 Correlation between TVPG and desaturation rate on $6 \mathrm{MWT}$ in $70 \mathrm{HP}$ patients. 6MWD, six minutes walking distance; HP, hypersensitivity pneumonitis. 


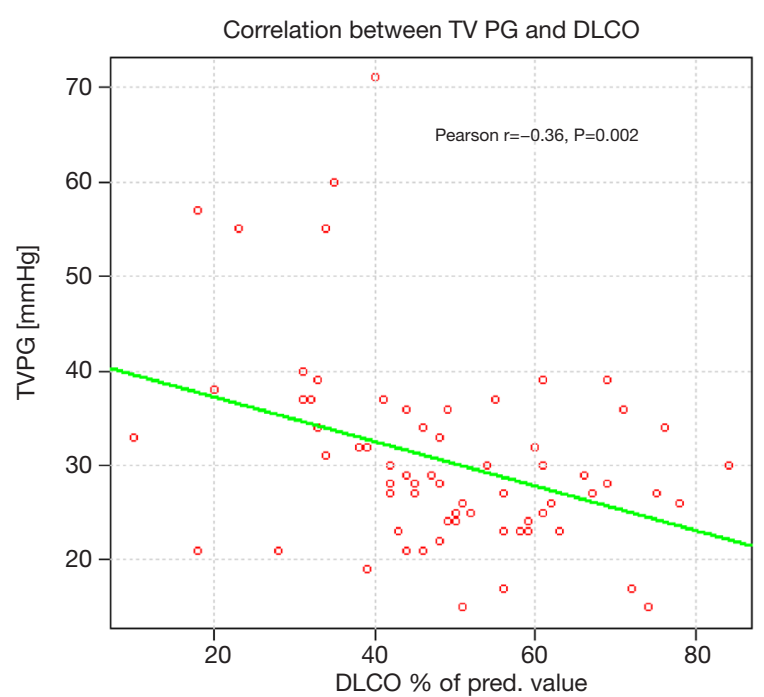

Figure 3 Correlation between TVPG and TLCO in $70 \mathrm{HP}$ patients. TLCO, lung transfer capacity for carbon monoxide; HP, hypersensitivity pneumonitis.

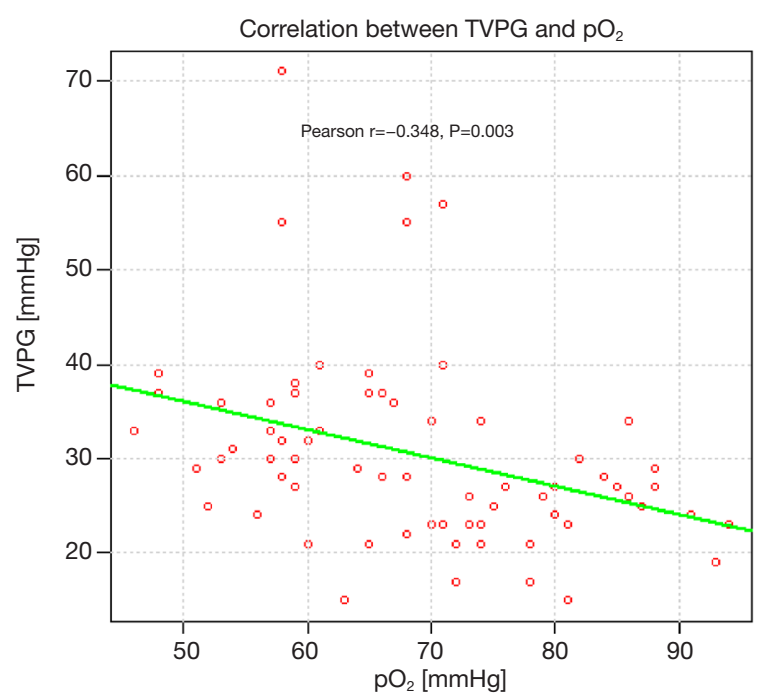

Figure 4 Correlation between TVPG and $\mathrm{PaO}_{2}$ in $70 \mathrm{HP}$ patients. $\mathrm{PaO}_{2}$, partial oxygen tension; HP, hypersensitivity pneumonitis.

patients with sarcoidosis (16). ARTEMIS-IPF study confirmed the presence of Group $3 \mathrm{PH}$ on RHC in 14\% of IPF patients with mild-to moderate restriction (17).

However, there is not much data in literature, concerning $\mathrm{PH}$ prevalence, in HP. The first report was published in 2012 by Koschel et al., who found PASP $>50 \mathrm{mmHg}$ on echocardiography in $19 \%$ of fibrotic HP patients (18). The epidemiological study of Walscher et al. published recently, documented $\mathrm{PH}$ in $9.5 \%$ of consecutive HP patients, but $\mathrm{PH}$ definition was based on medical diagnosis only (19).

We found PASP $>36 \mathrm{mmHg}$ in $37 \%$ of $\mathrm{HP}$ patients at diagnosis, and PASP $>50 \mathrm{mmHg}$ in $7 \%$. PASP $>50 \mathrm{mmHg}$ was diagnosed exclusively in fibrotic HP, and concerned $11 \%$ of the group.

Thus, based on our observations, and the data of Koschel et al. (18), we presumed, that highly probable diagnosis of $\mathrm{PH}$ on echocardiography (PASP $>50 \mathrm{mmHg}$ ) may concern $11-19 \%$ of patients with fibrotic HP at diagnosis.

The only report concerning hemodynamic evaluation of $\mathrm{PH}$ in HP was published by Oliveira et al., who documented $\mathrm{mPAP} \geq 25 \mathrm{mmHg}$ in 25 out of 50 consecutive patients with fibrotic HP (50\%), but severe PH (mPAP $>35 \mathrm{mmHg}$ ) was noted in $10 \%$ only (20).

The discrepancies between researchers concerning $\mathrm{PH}$ frequency in HP, could have been caused by different characteristics of study groups, e.g., the number of patients with fibrotic HP included, time from disease onset to diagnosis, and the patients' age. Nevertheless, time from first symptoms to diagnosis was not significantly different in our group of patients with PASP $>36 \mathrm{mmHg}$, comparing to those with PASP $\leq 36 \mathrm{mmHg}$. Likewise, no significant differences of patients age at diagnosis were noted between the two groups.

Clinical profile of our HP patients with PASP $>36 \mathrm{mmHg}$, showed significantly decreased exercise capacity (decreased walking distance, increased desaturation rate) and significantly lower resting $\mathrm{PaO}_{2}$ comparing to those with PASP $\leq 36 \mathrm{mmHg}$. This was the result of impaired lung diffusion capacity. Mean TLCO $\%$ pred. was $41.08 \%$ in those with PASP $>36 \mathrm{mmHg}$ and $52.64 \%$ pred. in the remaining ones.

It is worth to notice that despite relatively mild decrease of mean lung volumes in our study group (TLC $88.58 \%$ pred., VC $81.52 \%$ pred.), mean TLCO $\%$ pred. was disproportionally low (48\% pred.). According to the opinion of pulmonary specialists in DELPHI study, low TLCO at diagnosis in the patient with ILD presenting with HRCT signs of small airways disease, is very suggestive of HP diagnosis (21). The other authors documented that significant TLCO increase may be achieved during immunosuppressive therapy in HP patients, resulting in the large clinical benefit $(22,23)$.

In our study group, optimal predictors of $\mathrm{PH}$ on echocardiography, were $\mathrm{PaO}_{2}<69 \mathrm{mmHg}, 6 \mathrm{MWD}<455$ meters, TLCO $<42 \%$ pred. and $6 \mathrm{MWT}$ desaturation rate $>8 \%$. 
Table 4 HRCT findings in HP patients with echocardiographic signs of PH comparing to those without the echocardiographic signs of PH

\begin{tabular}{|c|c|c|c|c|c|c|c|}
\hline HRCT type of changes/intensity & \multicolumn{3}{|c|}{ PASP > 36 mmHg 27 cases, $n$} & \multicolumn{3}{|c|}{ PASP $\leq 36$ mmHg 43 cases, $n$} & $\mathrm{P}$ \\
\hline Centrilobular nodules & 17 & 8 & 2 & 22 & 15 & 6 & 0.56 \\
\hline Ground glass opacities & 5 & 12 & 10 & 8 & 22 & 13 & 0.82 \\
\hline Mosaic lung attenuation & 5 & 12 & 10 & 7 & 22 & 14 & 0.86 \\
\hline
\end{tabular}

HRCT, high resolution chest computed tomography; HP, hypersensitivity pneumonitis; PH, pulmonary hypertension; PASP, pulmonary artery systolic pressure.

Table 5 The utility of various parameters for prediction of $\mathrm{PH}$ on echocardiography in $70 \mathrm{HP}$ patients—ROC analysis

\begin{tabular}{|c|c|c|c|c|c|c|c|c|}
\hline Parameter & $A \cup C$ & $95 \% \mathrm{Cl}$ & $\mathrm{P}$ & Optimal cut off & Spec & Sens & PPV & NPV \\
\hline TLCO (\% pred.) & 0.723 & $0.587-0.723$ & 0.037 & $42 \%$ & 0.89 & 0.62 & 0.76 & 0.80 \\
\hline 6MWD meters & 0.726 & $0.603-0.726$ & 0.026 & 455 meters & 0.8 & 0.69 & 0.67 & 0.81 \\
\hline 6MWT desat (\%) & 0.732 & $0.608-0.732$ & 0.027 & $8 \%$ & 0.77 & 0.58 & 0.6 & 0.76 \\
\hline VC/DLCO & 0.636 & $0.499-0.636$ & 0.21 & 1.69 & 0.59 & 0.62 & 0.47 & 0.72 \\
\hline
\end{tabular}

$\mathrm{PH}$, pulmonary hypertension; HP, hypersensitivity pneumonitis; ROC, receiver operator characteristic curve; AUC, area under the curve; spec, specificity; sens, sensitivity; PPV, positive predictive value; NPV, negative predictive value; $\mathrm{PaO}_{2}$, partial oxygen tension measured in arterialized capillary blood; TLCO, lung transfer capacity for carbon monoxide; 6MWD, six minutes walking test distance; 6MWT, six minutes walking test; TLC, total lung capacity.

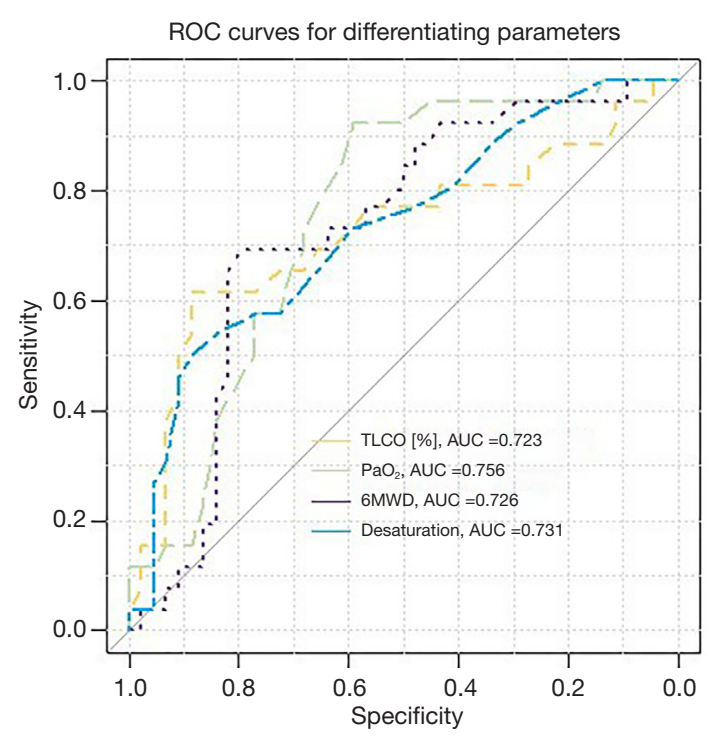

Figure 5 Diagnostic utility of various parameters for prediction of PASP $>36 \mathrm{mmHg}$ on echocardiography in $70 \mathrm{HP}$ patientsROC analysis. PASP, pulmonary artery systolic pressure; HP, hypersensitivity pneumonitis; ROC, receiver operator characteristic curve.
TLCO $<42 \%$ pred. increased probability of PASP $>36 \mathrm{mmHg}$ by five-fold, and $6 \mathrm{MWT}$ desaturation rate $>8 \%$ by four-fold.

According to Oliveira et al. $\mathrm{PaO}_{2}$ and FVC were the independent predictors of $\mathrm{PH}$ at RHC, nevertheless, this group was composed of patients with much more advanced lung disease, mean FVC was 59\% pred. (20).

We previously found FVC/TLCO index and TLC/ TLCO index as significant predictors of $\mathrm{PH}$ on echocardiography in the group of 93 patients with various interstitial lung diseases (ILDs), nevertheless we failed to demonstrate the utility of these parameters in $\mathrm{PH}$ prediction in the present group of patients (24).

What may be interesting, NT-proBNP serum level was not helpful in $\mathrm{PH}$ prediction, neither in our study group, nor in the study of Oliveira et al. (20). According to our observations, serum NT-proBNP increase is a late sequel of right heart dysfunction in group $3 \mathrm{PH}$, thus it is not helpful in $\mathrm{PH}$ screening at the time of ILDs recognition.

We are the first authors who investigated the relationship 
Table 6 Risk of $\mathrm{PH}$ on echocardiography—univariate analysis

\begin{tabular}{lcccc}
\hline Variable & Cut off & $\mathrm{RR}$ & $95 \% \mathrm{Cl}$ & $\mathrm{P}$ \\
\hline TLCO (\% pred.) & 42 & 5.42 & $2.82-7.43$ & 0.00005 \\
$\mathrm{PaO}_{2}(\mathrm{mmHg})$ & 69 & 2.22 & $1.57-2.56$ & 0.00069 \\
$6 \mathrm{MWD}$ (meters) & 455 & 3.38 & $2.08-4.26$ & 0.00013 \\
6MWT desat \% & 8 & 4.4 & $2.03-6.77$ & 0.00085 \\
\hline
\end{tabular}

$\mathrm{PH}$, pulmonary hypertension; TLCO, lung transfer capacity for carbon monoxide; $\mathrm{PaO}_{2}$, partial oxygen tension measured in arterialized capillary blood; 6MWD, six minutes walking test distance; $6 \mathrm{MWT}$, six minutes walking test; RR, relative risk.

between lung fibrosis and echocardiographic signs of PH. HRCT analysis revealed that fibrotic HP was equally frequent among patients with PASP $>36 \mathrm{mmHg}$ and those with PASP $\leq 36 \mathrm{mmHg}$. Moreover 9 patients without lung fibrosis had PASP $\geq 36 \mathrm{mmHg}$, and 10 patients with advanced lung fibrosis (honeycombing) had no echocardiographic signs of $\mathrm{PH}$. Thus, lung fibrosis was not a good predictor of $\mathrm{PH}$ on echocardiography, if PASP of $36 \mathrm{mmHg}$ was considered as cut off value. Nevertheless, PASP $>50 \mathrm{mmHg}$ was observed exclusively among fibrotic $\mathrm{HP}$ patients. Unfortunately, PASP $>50 \mathrm{mmHg}$ was a rare finding in our study group (7\%), despite the fact that fibrotic lung disease was frequent (64\% of patients). Therefore the role of lung fibrosis as $\mathrm{PH}$ predictor has not been confirmed.

The limitations of our study concerned mostly its' retrospective character. Our clinical practice is to perform echocardiographic examination in HP at diagnosis, but occasionally, initial echocardiography was lacking due to technical reasons. Therefore some of the patients diagnosed with HP in between 2005 and 2017, were not included into the present study. Moreover patients with echocardiographic PASP $>50 \mathrm{mmHg}$, RHC was not performed to confirm PH diagnosis. Nevertheless this concerned only 5 patients, and in 3 of them-advanced fibrotic HP, with honeycombingwas diagnosed.

\section{Conclusions}

We found echocardiographic signs of $\mathrm{PH}$ in $37 \%$ of $\mathrm{HP}$ patients at diagnosis, but most of them presented with moderately increased PASP. PH probability was significantly increased in patients with $\mathrm{PaO}_{2}<69 \mathrm{mmHg}$, TLCO $<42 \%$ pred., $6 \mathrm{MWD}<455$ meters, and desaturation rate exceeding $8 \%$. The presence of lung fibrosis on HRCT, the duration of the disease, and the patients' age at diagnosis, were not helpful in prediction of $\mathrm{PH}$ on echocardiography.

\section{Acknowledgments}

Funding: The work was supported by National Tuberculosis and Lung Diseases Research Institute, statutory project No. 7.48 .

\section{Footnote}

Reporting Checklist: The authors have completed the STARD reporting checklist. Available at https://dx.doi. org/10.21037/jtd-21-130

Data Sharing Statement: Available at https://dx.doi. org/10.21037/jtd-21-130

Peer Review File: Available at https://dx.doi.org/10.21037/ jtd-21-130

Conflicts of Interest: All authors have completed the ICMJE uniform disclosure form (available at https://dx.doi. org/10.21037/jtd-21-130). The authors have no conflicts of interest to declare.

Ethical Statement: The authors are accountable for all aspects of the work in ensuring that questions related to the accuracy or integrity of any part of the work are appropriately investigated and resolved. The study was conducted in accordance with the Declaration of Helsinki (as revised in 2013). The study was approved by Bioethical Committee, National Tuberculosis and Lung Diseases Research Institute (NP.: 14/2019) and informed consent was taken from all the patients.

Open Access Statement: This is an Open Access article distributed in accordance with the Creative Commons Attribution-NonCommercial-NoDerivs 4.0 International License (CC BY-NC-ND 4.0), which permits the non- 
commercial replication and distribution of the article with the strict proviso that no changes or edits are made and the original work is properly cited (including links to both the formal publication through the relevant DOI and the license). See: https://creativecommons.org/licenses/by-nc-nd/4.0/.

\section{References}

1. Vasakova M, Morell F, Walsh S, et al. Hypersensitivity pneumonitis: perspectives in diagnosis and management. Am J Respir Crit Care Med 2017;196:680-9.

2. Raghu G, Remy-Jardin M, Ryerson J, et al. Diagnosis of hypersensitivity pneumonitis in adults. An Official ATS/ JRS/ALAT Clinical Practice Guideline. Am J Respir Crit Care Med 2020;202:e36-69.

3. Pedraza-Serrano F, Jimenez-Garcia R, Lopez-de-Andres A, et al. Characteristics and outcomes of patients hospitalized with interstitial lung diseases in Spain, 2014 to 2015. Medicine 2019;98:e15779.

4. Fisher JH, Kolb M, Algamdi M, et al. Baseline characteristics and comorbidities in the Canadian Registry for pulmonary fibrosis. BMC Pulm Med 2019;19:223.

5. Rittig AH, Hilberg $\mathrm{O}$, Ibsen $\mathrm{R}$, et al. Incidence, comorbidity and survival rate of hypersensitivity pneumonitis:a national population-based study. ERJ Open Res 2019;5:00259-2018.

6. Fernández Pérez ER, Kong AM, Raimundo K, et al. Epidemiology of hypersensitivity pneumonitis among an insured population in the United States: a claims-based cohort analysis. Ann Am Thorac Soc 2018;15:460-9.

7. Szturmowicz M, Barańska I, Jędrych ME, et al. Hypersensitivity pneumonitis recognised in a single pulmonary unit, between 2005 and 2015: comparison with recently proposed diagnostic criteria. Adv Respir Med 2019;87:83-9.

8. Szturmowicz M, Garczewska B, Jędrych ME, et al. The value of serum precipitins against specific antigens in patients diagnosed with hypersensitivity pneumonitis retrospective study. Cent Eur J Immunol 2019;44:390-4.

9. Galiè N, Hoepper MM, Humbert M, et al. Guidelines for the diagnosis and treatment of pulmonary hypertension. Eur Respir J 2009;34:1219-63.

10. Pellegrino R, Viegi G, Brusasco V, et al. Interpretative strategies for lung function test. Eur Respir $\mathrm{J}$ 2005;26:948-68.
11. Quanjer PH, Tammeling G, Cotes J, et al. Lung volumes and forced ventilator flows. Report Working party. Standardization of lung function tests. European Community for Steel and Coal. Eur Respir J 1993;6:5-40.

12. Falaschetti E, Laiho J, Primatesta P, et al. Prediction equations for normal and low lung function from the Health Survey for England. Eur Respir J 2004;23:456-63.

13. Macintyre N, Crapo RO, Viegi G, et al. Standardization of the single-breath determination of carbon monoxide uptake in the lung. Eur Respir J 2005;26:720-35.

14. ATS Committee on proficiency standards for clinical function laboratories. ATS statement: Guidelines for six-minute walk test:Am J Respir Crit Care Med 2002;166:111-7.

15. Nathan SD, Barbera JA, Gaine SP, et al. Pulmonary hypertension in chronic lung disease and hypoxia. Eur Respir J 2019;53:1801914.

16. Huitema MP, Bakker A, Mager J, et al. Prevalence of pulmonary hypertension in pulmonary sarcoidosis: the first large European prospective study. Eur Respir J 2019;54:1900897.

17. Raghu G, Nathan SD, Behr J, et al. Pulmonary hypertension in idiopathic pulmonary fibrosis with mild-to moderate restriction. Eur Respir J 2015;46:1370-7.

18. Koschel DS, Cardoso C, Wiedemann B. Pulmonary hypertension in chronic hypersensitivity pneumonitis. Lung 2012;190:295-302.

19. Wälscher J, Gross B, Morisset J, et al. Comorbidities and survival in patients with chronic hypersensitivity pneumonitis. Respir Res 2020;21:12.

20. Oliveira RKF, Pereira CAC, Ramos RP, et al. A hemodynamic study of pulmonary hypertension in chronic hypersensitivity pneumonitis. Eur Respir J 2014;44:415-24.

21. Morisset J, Johannson KA, Jones KD, et al. Identification of diagnostic criteria for chronic hypersensitivity pneumonitis. An International Modified Delphi Survey. Am J Respir Crit Care Med 2018;197:1036-44.

22. Morisset J, Johannson KA, Vittinghoff E, et al. Use of mycophenolate mofetil or azathioprine for the management of chronic hypersensitivity pneumonitis. Chest 2017;151:619-25.

23. Fiddler CA, Simler N, Thillai M, et al. Use of mycophenolate mofetil and azathioprine for the treatment of chronic hypersensitivity pneumonitis - A single-centre 
experience. Clin Respir J 2019;13:791-4.

24. Sobiecka M, Lewandowska K, Kober J, et al. Can a new scoring system improve prediction of pulmonary hypertension in newly recognised interstitial lung diseases? Lung 2020;198:547-54.

Cite this article as: Dybowska $M$, Barańska I, Franczuk M, Skoczylas A, Szturmowicz M. Echocardiographic signs of pulmonary hypertension in patients with newly recognized hypersensitivity pneumonitis, prevalence and clinical predictors. J Thorac Dis 2021;13(7):3988-3997. doi: 10.21037/jtd-21-130 